\title{
Optical Modulation Format Recognition in Stokes Space for Digital Coherent Receivers
}

Borkowski, Robert; Zibar, Darko; Caballero Jambrina, Antonio; Arlunno, Valeria; Tafur Monroy, Idelfonso

Published in:

2013 Optical Fiber Communication Conference and Exposition and the National Fiber Optic Engineers Conference (OFC/NFOEC)

Link to article, DOI:

10.1364/OFC.2013.OTh3B.3

Publication date:

2013

Link back to DTU Orbit

Citation (APA):

Borkowski, R., Zibar, D., Caballero Jambrina, A., Arlunno, V., \& Tafur Monroy, I. (2013). Optical Modulation Format Recognition in Stokes Space for Digital Coherent Receivers. In 2013 Optical Fiber Communication Conference and Exposition and the National Fiber Optic Engineers Conference (OFC/NFOEC) (pp. OTh3B.3). IEEE. https://doi.org/10.1364/OFC.2013.OTh3B.3

\section{General rights}

Copyright and moral rights for the publications made accessible in the public portal are retained by the authors and/or other copyright owners and it is a condition of accessing publications that users recognise and abide by the legal requirements associated with these rights.

- Users may download and print one copy of any publication from the public portal for the purpose of private study or research.

- You may not further distribute the material or use it for any profit-making activity or commercial gain

- You may freely distribute the URL identifying the publication in the public portal 


\title{
Optical Modulation Format Recognition in Stokes Space for Digital Coherent Receivers
}

\author{
Robert Borkowski, Darko Zibar, Antonio Caballero, Valeria Arlunno and Idelfonso Tafur Monroy \\ DTU Fotonik - Department of Photonics Engineering, Technical University of Denmark, Oersteds Plads 343, 2800 Kgs. Lyngby, Denmark \\ rbor@fotonik.dtu.dk
}

\begin{abstract}
We report on a novel method for optical modulation format recognition based on Stokes parameters and variational expectation maximization algorithm. Discrimination among six different pol-muxed coherent modulation formats is successfully demonstrated in simulation and experiment.

OCIS codes: (060.1660) Coherent communications; (060.4080) Modulation
\end{abstract}

\section{Introduction}

Transponders and networks offering flexibility by adaptation of the modulation format are currently under intensified research and even available commercially [1]. In general case, it cannot be guaranteed any longer that signals arriving at a specific receiver unit will have the same, known in advance, modulation format. Adaptation of the transmitter may be performed on-the-fly and this creates a need for complementary techniques on the receiver side. Modulation format recognition (MFR) [2] is essential to ensure that signals are properly demodulated regardless of the modulation used by the transmitter. This is one of the enabling methods for a software defined receiver (SDR) and is implemented in a digital signal processing (DSP) circuit of a digital coherent receiver.

The topic of MFR has been well explored for radio communications [3]. Nonetheless, MFR for fiber optic communications still remains underdeveloped. In one paper, $k$-means algorithm is used, which, however, requires carrier and phase recovery prior to modulation classification [4]. In another publication, artificial neural network that needs prior training are used [5].

In this paper we propose a novel MFR method based on Stokes space measurements and variational Bayesian expectation maximization (VBEM) algorithm [6]. It is a significant improvement over the constellation analysisbased techniques, such as $k$-means (comp. Fig. 1) and allows for MFR at a considerably earlier stage, enabling optimization of follow-up DSP algorithms depending on the detected modulation format. One example might be a decision directed equalization and carrier recovery for which the knowledge of the ideal constellation is essential. The method is insensitive to polarization mixing, carrier frequency offset and phase offset and does not require training. We perform numerical and experimental validation and report on a successful modulation classification by discriminating from a possible set of BPSK, QPSK, 8-PSK, 8-QAM, 12-QAM and 16-QAM modulations. The method is targeted at digital coherent receivers; nonetheless, it may be employed in any receiver capable of measuring Stokes parameters.

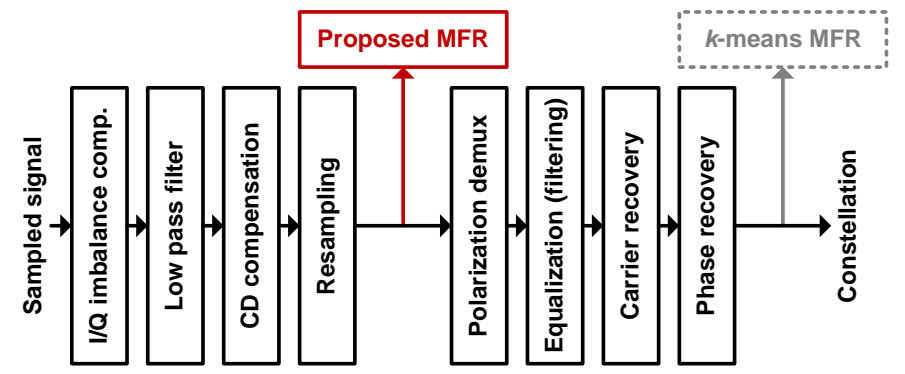

Fig. 1. Placement of the proposed MFR block in the receivers' DSP chain and comparison to another MFR method based on $k$-means [4].

\section{Proposed MFR technique}

Assuming $x$ and $y$ are two received orthogonal complex optical fields, the Stokes parameters are given as $S_{0}=|x|^{2}+|y|^{2}, \quad S_{1}=|x|^{2}-|y|^{2}, \quad S_{2}=2 \mathfrak{R}(x \bar{y}), \quad S_{3}=2 \mathfrak{I}(x \bar{y})$. Points inside a 3-dimensional (3D) sphere of the Stokes space are triplets $\left(S_{1}, S_{2}, S_{3}\right)$ obtained by transforming the received signal samples according to those equations. Stokes parameters of any polarization division multiplexed (PDM) coherent optical modulation format are bounded by a lens shape contained within the sphere [7]. Each modulation format has a different signature in the Stokes space. Signatures of PDM modulation formats under consideration - \{BPSK, QPSK, 8-PSK, 8-QAM, 12-QAM, 16-QAM $\}$ - are shown in Fig 3. Those modulation formats result in, respectively, $N_{d e t}=\{2,4,8,16,32,60\}$ clusters (point clouds) in the Stokes space. This feature is used to discriminate between different modulation formats 
and ultimately perform MFR. By applying VBEM for Gaussian mixture models (GMM) [6] it is possible to determine the number of clusters. The expectation maximization algorithm iteratively finds maximum likelihood for parameters of probability distributions created by transformation of the signal to the Stokes space. Since GMM was used, the set of parameters to optimize were means and covariances of 3D Gaussian distributions. It is necessary to perform this step as polarization mixing may lead to arbitrary rotation of the received signature around the origin of the Stokes space [7]. A variational treatment is necessary in order to discover the actual number of clusters of the mixture. The model is initialized with a large number of clusters $\left(N_{\text {init }}=924\right)$ that well exceeds the actual number to be detected $\left(\max \left(N_{\text {det }}\right)=60\right.$ for 16-QAM $)$. The concentration parameters $\alpha_{k}$ of the Dirichlet distribution for each cluster $k=0 \ldots N-1$ are in the beginning the same, meaning that each cluster has the same importance. After iterating the model, the concentration parameters are updated with only a few of them having significant values - denoting the 'surviving' clusters. The remaining clusters are either negligibly small or nonexistent in the posterior. The concentration parameters were used to construct a following cost function $j_{N}$, defined as $j_{N}=\left(\frac{1}{N} \sum_{k=0}^{N-1} \alpha_{k}\right) /\left(\frac{1}{N_{\text {in } i t}-N} \sum_{k=N}^{N_{\text {init }}-1} \alpha_{k}\right), N \in N_{\text {det }}$ and normalized cost function $J_{N}=j_{N} / \sum_{N} j_{N}$. The normalized cost function sums up to one over all $N$ and may be interpreted as a probability that the investigated data form $N$ distinct clusters in the Stokes space. Since $N$ is a bijective function of the modulation formats from among which discrimination is made, unambiguous modulation classification can be performed.

\section{Simulation and experiment setup}

To demonstrate the feasibility of our approach a numerical simulation is performed involving all modulation formats from among which discrimination can be made. $10^{4} \cdot \log _{2} M$ points, where $M$ is the modulation order, of a 10 Gbaud PDM signal were received through an additive white Gaussian noise channel. Noise power was varied to maintain signal-to-noise ratio (SNR) at a level of $30 \mathrm{~dB}$ for all modulation formats. Next, a carrier frequency offset and phase offset is applied. Finally VBEM algorithm is run and the modulation format is recognized.

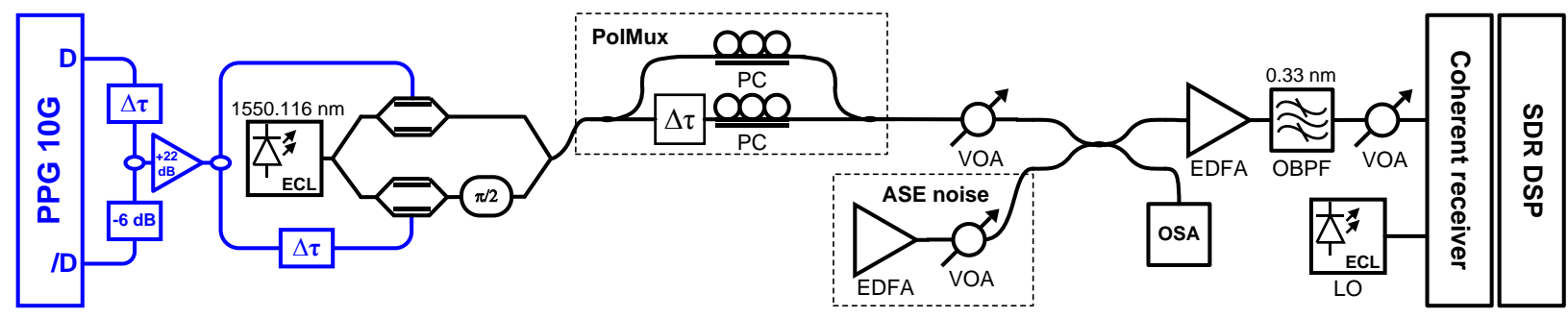

Fig. 2. Experimental setup. PPG: pulse patter generator, ECL: external cavity laser, PC: polarization controller, EDFA: erbium-doped fiber amplifier, VOA: variable optical attenuator, OBPF: optical bandpass filter, LO: local oscillator.

The proposed idea was subsequently validated in an experiment. The employed experimental setup, including reconfigurable PDM 16-QAM and PDM QPSK generation stage at 10 Gbaud, is presented in Fig. 2. $100 \mathrm{kHz}-$ linewidth laser centered at $1550.116 \mathrm{~nm}$ was used as a carrier signal and passed to an optical I/Q modulator. The I/Q modulator was driven with either 4-level or 2-level electrical signal, depending on whether /D output of a $10 \mathrm{~Gb} / \mathrm{s}$ pulse pattern generator (PPG) was, respectively, enabled or disabled. In the optical domain this resulted in 16-QAM or QPSK signals, later polarization multiplexed to create PDM 16-QAM at $80 \mathrm{~Gb} / \mathrm{s}$ or PDM QPSK at $40 \mathrm{~Gb} / \mathrm{s}$. The signal was received back-to-back and amplified spontaneous emission (ASE) noise was added by an ASE noise stage. Optical SNR of $27 \mathrm{~dB}$ for 16-QAM and $19 \mathrm{~dB}$ for QPSK was set. At the receiver side, the signal was filtered by a $0.33 \mathrm{~nm}$-broad optical bandpass filter (OBPF) to remove excess of ASE noise before digitization. A preamplifier and variable optical attenuator (VOA) was used in order to keep the power entering the front-end of a coherent receiver at a constant level, in the optimal operating point. Another $100 \mathrm{kHz}-$ linewidth laser, offset by several hundred $\mathrm{MHz}$ from the carrier wavelength was used as a local oscillator signal (LO) and supplied to the coherent receiver. Electrical outputs of the coherent receiver were sampled at $40 \mathrm{GSa} / \mathrm{s}$ with a $13 \mathrm{GHz}$-bandwidth digital storage oscilloscope (DSO). Traces acquired from the DSO were processed offline by a set of SDR DSP algorithms capable of demodulating both 16-QAM and QPSK. VBEM was then run after digital resampling (Fig. 1).

\section{Results}

Fig. 3 show the outcome of VBEM with points belonging to each discovered cluster plotted in a different color. Stokes space plots and corresponding recovered constellations are shown in Fig. 3(a-f) for simulated cases and in Fig. 3(g-h) for experimental measurements. Fig. 4 shows the value of normalized cost functions for: (a) simulations and (b) measurements. Modulation formats among which discrimination was performed are on the horizontal axis. Each color denotes a different case in which the actual modulation format to be detected is defined in the legend. Bar height denotes value of the normalized cost function $J$, i.e. the measure of how certain the detection is. The recognition is performed by finding $N$ for which $J$ attains its maximum. Bars that indicate successful classification 
are marked by arrows. The results presented in Fig. 4 demonstrated that all modulation format classifications were performed successfully. The cost function for 16-QAM peaks around 0.8 while for other modulation formats around 0.5 . This follows from the definition of the cost function and its slow decay as modulations with $N$ higher than the actual are tested.

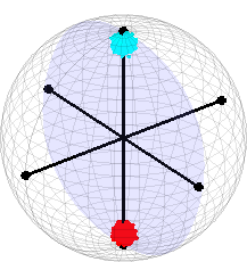

(a) PDM BPSK, 2 clusters/2 points

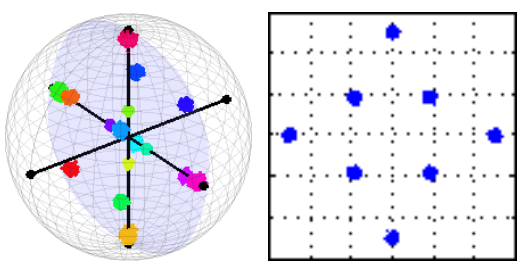

(d) PDM 8-QAM, 16 clusters/8 points

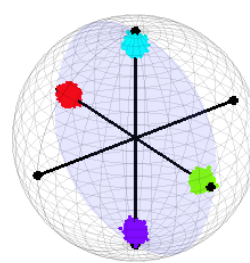

(b) PDM QPSK, 4 clusters/4 points

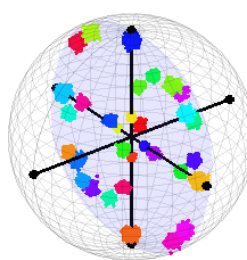

(e) PDM 12-QAM, 32 clusters/12 points

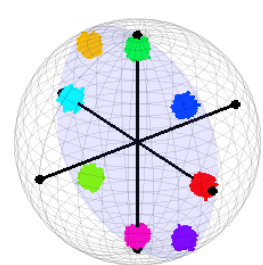

(c) PDM 8-PSK, 8 clusters/8 points
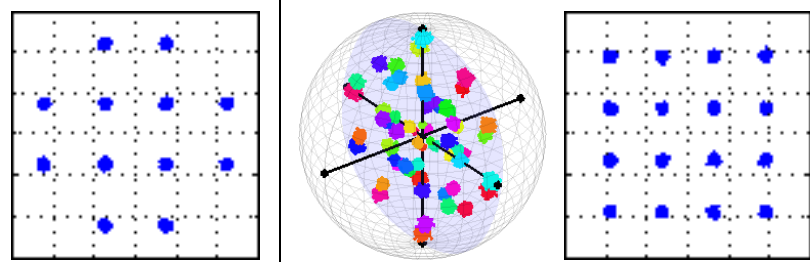

(f) PDM 16-QAM, 60 clusters/16 points

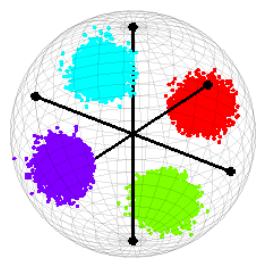

(g) experimental PDM QPSK

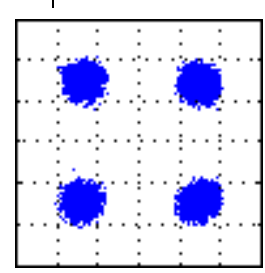

K
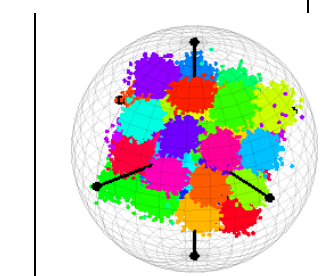

(h) experimental PDM 16-QAM

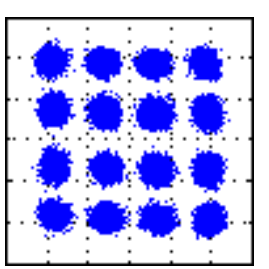

Fig. 3. Simulation (a-f) and experimental data ( $\mathrm{g}-\mathrm{h})$ for different modulation formats. Stokes space representation of the signal with the number of clusters (left) and corresponding recovered constellations with the modulation order (right) are shown.

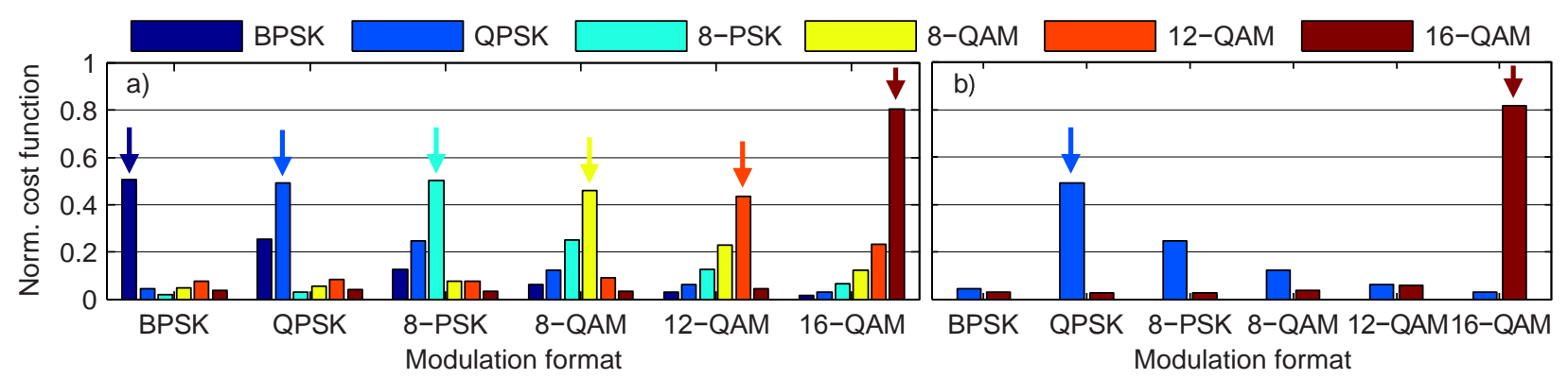

Fig. 4. Normalized cost function for (a) numerical simulation data and (b) experimental data. Arrows indicate bars that should be the tallest if classification was successful. It is shown that all investigated cases were successfully classified.

\section{Summary}

In this paper we propose a novel modulation format recognition method from Stokes space parameters using VBEM algorithm. The method is insensitive to polarization mixing, carrier frequency offset and phase offset and does not require training. The technique was used to successfully discriminate between PDM BPSK, QPSK, 8-PSK, 8-QAM, 12-QAM and 16-QAM in numerical simulation and recognize 16-QAM and QPSK from experimental data. The technique can be applied in any receiver capable of measuring Stokes parameters.

\section{References}

[1] K. Roberts, C. Laperle, “Flexible Transceivers,” in Proc. ECOC'12, (2012).

[2] I. T. Monroy et al., "Cognitive Heterogeneous Reconfigurable Optical Networks (CHRON): Enabling technologies and techniques," in Proc. ICTON'11, (2011).

[3] O. A. Dobre et al., "Survey of automatic modulation classification techniques: classical approaches and new trends," IET Commun. 1, 137-156 (2007).

[4] N. Guerrero Gonzalez et al., "Cognitive digital receiver for burst mode phase modulated radio over fiber links," in Proc. ECOC'10, (2010).

[5] F. N. Khan et al., "Modulation format identification in heterogeneous fiber-optic networks using artificial neural networks," Opt. Express 20, 12422-12431 (2012).

[6] C. M. Bishop, Pattern Recognition and Machine Learning, (Springer, 2006), $3^{\text {rd }}$ ed., ISBN 978-0-387-31073-2.

[7] B. Szafraniec et al., "Polarization demultiplexing in Stokes space," Opt. Express 18, 17928-17939 (2010). 